\title{
International publications of authors from Bosnia and Herzegovina in Current Contents indexed publications in the second half of $2016^{*}$
}

Amra S, O'Horo JC, Singh TD, Wilson GA, Kashyap R, Petersen R, Roberts RO, Fryer JD, Rabinstein AA, Gajic O. Derivation and validation of the automated search algorithms to identify cognitive impairment and dementia in electronic health records. J Crit Care. 2017 Feb;37:202-205. doi: 10.1016/j. jcrc.2016.09.026. Epub 2016 Oct 8.

Babić E, Bevanda M, Karin M, Volarić M, Bevanda D, Glibo DB, Bogut A. Correlation of clinical and endoscopic indices in IBD patients in University Clinical Hospital Mostar. Psychiatr Danub. 2016 Dec;28(Suppl-2):242-246.

Babić N, Dogan S, Čakar J, Pilav A, Marjanović D, Hadžiavdić V. Molecular diversity of 23 Y-chromosome short tandem repeat loci in the population of Tuzla Canton, Bosnia and Herzegovina. Ann Hum Biol. 2016 Nov 30:1-8. doi: 10.1080/03014460.2016.1259430. [Epub ahead of print]

Bell J, Ancillotti M, Coathup V, Coy S, Rigter T, Tatum T, Grewal J, Akcesme FB, Brkić J, CausevicRamosevac A, Milovanovic G, Nobile M, Pavlidis C, Finlay T, Kaye J; ELSI2.0. Challenges and opportunities for ELSI early career researchers. BMC Med Ethics. 2016 Jul 8;17(1):37. doi: 10.1186/s12910016-0121-5.

Biegstraaten M, Cox TM, Belmatoug N, Berger MG, Collin-Histed T, Vom Dahl S, Di Rocco M, Fraga C, Giona F, Giraldo P, Hasanhodzic M, Hughes DA, Iversen PO, Kiewiet AI, Lukina E, Machaczka M, Marinakis T, Mengel E, Pastores GM, Plöckinger U, Rosenbaum H, Serratrice C, Symeonidis A, Szer J, Timmerman J, Tylki-Szymańska A, Weisz Hubshman M, Zafeiriou DI, Zimran A, Hollak CE. Management goals for type 1 Gaucher disease: An expert consensus document from the European working group on Gaucher disease. Blood Cells Mol Dis. 2016 Oct 24. pii: S1079-9796(16)30191-7. doi: 10.1016/j. bcmd.2016.10.008. [Epub ahead of print]
Bjelica D, Idrizovic K, Popovic S, Sisic N, Sekulic D, Ostojic L, Spasic M, Zenic N. An Examination of the Ethnicity-Specific Prevalence of and Factors Associated with Substance Use and Misuse: Cross-Sectional Analysis of Croatian and Bosniak Adolescents in Bosnia and Herzegovina. Int J Environ Res Public Health. 2016 Sep 29;13(10). pii: E968.

Bobić B, Milosavić M, Guzijan G, DjurkovićDjaković O. First Report on Toxoplasma gondii Infection in Bosnia and Herzegovina: Study in Blood Donors. Vector Borne Zoonotic Dis. 2016 Dec;16(12):807-809. Epub 2016 Oct 31.

Bohac M, Anticic M, Draca N, Kozomara B, Dekaris I, Gabric N, Patel S. Comparison of Verisyse and Veriflex Phakic Intraocular Lenses for Treatment of Moderate to High Myopia 36 Months after Surgery. Semin Ophthalmol. 2016 Aug 3:1-9. [Epub ahead of print]

Buhovac M. Crimes against Public Health in Croatia and Bosnia and Herzegovina. Psychiatr Danub. 2016 Dec;28(Suppl-2):209-215.

Burnazovic-Ristic L, Rakanovic-Todic M, Maleskic S, Cesic AK, Kusturica J. Are we Scared of Clinical Trials if not Sufficiently Informed and Educated? Clin Ther. 2016 Oct 6;38(10S):e4-e5. doi: 10.1016/j. clinthera.2016.07.025.

Carreras-Torres R, Ferran A, Zanetti D, Esteban E, Varesi L, Pojskic N, Coia V, Chaabani H, Via $\mathrm{M}$, Moral P. Population structure from NOS genes correlates with geographical differences in coronary incidence across Europe. Am J Phys Anthropol. 2016 Dec;161(4):634-645. doi: 10.1002/ajpa.23063. Epub 2016 Aug 8.

Cenko E, Ricci B, Kedev S, Kalpak O, Câlmâc L, Vasiljevic Z, Knežević B, Dilic M, Miličić D, Manfrini O, Koller A, Dorobantu M, Badimon L, Bugiardini $\mathrm{R}$. The no-reflow phenomenon in the young and in

${ }^{\star}$ Data for this survey were collected from PubMed/MEDLINE using the keywords Bosnia and Herzegovina and 2016. 
the elderly. Int J Cardiol. 2016 Nov 1;222:1122-8. doi: 10.1016/j.ijcard.2016.07.209. Epub 2016 Aug 2.

Cenko E, Ricci B, Kedev S, Vasiljevic Z, Dorobantu M, Gustiene O, Knežević B, Miličić D, Dilic M, Manfrini O, Koller A, Badimon L, Bugiardini R. Invasive versus conservative strategy in acute coronary syndromes: The paradox in women's outcomes. Int J Cardiol. 2016 Nov 1;222:1110-5. doi: 10.1016/j.ijcard.2016.07.211. Epub 2016 Jul 30.

Čehić E, Kasum M, Šimunić V, Orešković S, Vujić G, Grgić F. Fertility in men with spinal cord injury. Gynecol Endocrinol. 2016 Dec;32(12):937-941. Epub 2016 Jul 16.

Delibegović S, Mehmedović Z. The Influence of the Appendiceal Base Diameter on Appendix Stump Closure in Laparoscopic Appendectomy. World J Surg. 2016 Oct;40(10):2342-7. doi: 10.1007/s00268016-3564-4.

Delibegović S, Mehmedović Z. Erratum to: The Influence of the Appendiceal Base Diameter on Appendix Stump Closure in Laparoscopic Appendectomy. World J Surg. 2016 Nov;40(11):2832.

Dogan S, Babic N, Gurkan C, Goksu A, Marjanovic D, Hadziavdic V. Y-chromosomal haplogroup distribution in the Tuzla Canton of Bosnia and Herzegovina: A concordance study using four different in silico assignment algorithms based on Y-STR data. Homo. 2016 Dec;67(6):471-483. doi: 10.1016/j.jchb.2016.10.003. Epub 2016 Nov 1.

Dujic T, Zhou K, Tavendale R, Palmer CN, Pearson ER. Effect of Serotonin Transporter 5-HTTLPR Polymorphism on Gastrointestinal Intolerance to Metformin: A GoDARTS Study. Diabetes Care. 2016 Nov;39(11):1896-1901. Epub 2016 Aug 4.

Dujic T, Zhou K, Yee SW, van Leeuwen N, de Keyser CE, Javorský M, Goswami S, Zaharenko L, Hougaard Christensen MM, Out M, Tavendale R, Kubo M, Hedderson MM, van der Heijden AA, Klimčáková L, Pirags V, Kooy A, Brøsen K, Klovins J, Semiz S, Tkáč I, Stricker BH, Palmer C, ‘t Hart LM, Giacomini KM, Pearson ER. Variants in pharmacokinetic transporters and glycemic response to metformin: A metgen meta-analysis. Clin Pharmacol Ther. 2016 Nov 10. doi: 10.1002/cpt.567. [Epub ahead of print]

Dussault MC, Smith M, Hanson I. Evaluation of trauma patterns in blast injuries using multiple correspondence analysis. Forensic Sci Int. 2016 Oct;267:66-72. doi: 10.1016/j.forsciint.2016.08.004. Epub 2016 Aug 11.
Dzinic SH, Bernardo MM, Li X, Fernandez-Valdivia R, Ho YS, Mi QS, Bandyopadhyay S, Lonardo F, Vranic S, Oliveira DS, Bonfil RD, Dyson G, Chen K, Omerovic A, Sheng X, Han X, Wu D, Bi X, Cabaravdic D, Jakupovic U, Wahba M, Pang A, Harajli D, Sakr WA, Sheng S. An Essential Role of Maspin in Embryogenesis and Tumor Suppression. Cancer Res. 2017 Feb 15;77(4):886-896. doi: 10.1158/0008-5472.CAN-16-2219. Epub 2016 Dec 6.

EAS Familial Hypercholesterolaemia Studies Collaboration, Vallejo-Vaz AJ, Akram A, Kondapally Seshasai SR, Cole D, Watts GF, Hovingh GK, Kastelein JJ, Mata P, Raal FJ, Santos RD, Soran $\mathrm{H}$, Freiberger T, Abifadel M, Aguilar-Salinas CA, Alnouri F, Alonso R, Al-Rasadi K, Banach M, Bogsrud MP, Bourbon M, Bruckert E, Car J, Ceska R, Corral P, Descamps O, Dieplinger H, Do CT, Durst R, Ezhov MV, Fras Z, Gaita D, Gaspar IM, Genest J, Harada-Shiba M, Jiang L, Kayikcioglu M, Lam CS, Latkovskis G, Laufs U, Liberopoulos E, Lin J, Lin N, Maher V, Majano N, Marais AD, März W, Mirrakhimov E, Miserez AR, Mitchenko O, Nawawi H, Nilsson L, Nordestgaard BG, Paragh G, Petrulioniene Z, Pojskic B, Reiner Ž, Sahebkar A, Santos LE, Schunkert H, Shehab A, Slimane MN, Stoll M, Su TC, Susekov A, Tilney M, Tomlinson B, Tselepis AD, Vohnout B, Widén E, Yamashita S, Catapano AL, Ray KK. Pooling and expanding registries of familial hypercholesterolaemia to assess gaps in care and improve disease management and outcomes: Rationale and design of the global EAS Familial Hypercholesterolaemia Studies Collaboration. Atheroscler Suppl. 2016 Dec;22:1-32. doi: 10.1016/j.atherosclerosissup.2016.10.001. Epub 2016 Dec 7.

Fabijanovic D, Zunic I, Martic TN, Skenderi F, Serman L, Vranic S. The expression of SFRP1, SFRP3, DVL1, and DVL2 proteins in testicular germ cell tumors. APMIS. 2016 Nov;124(11):942-949. doi: 10.1111/apm.12588. Epub 2016 Sep 7.

Foix MP, Dunatov A, Martinek P, Mundó EC, Suster S, Sperga M, Lopez JI, Ulamec M, Bulimbasic S, Montiel DP, Alaghehbandan R, Peckova K, Pivovarcikova K, Ondrej D, Rotterova P, Skenderi F, Prochazkova K, Dusek M, Hora M, Michal M, Hes O. Morphological, immunohistochemical, and chromosomal analysis of multicystic chromophobe renal cell carcinoma, an architecturally unusual challenging variant. Virchows Arch. 2016 Dec;469(6):669-678. Epub 2016 Sep 15.

Gatalica Z, Vranic S, Xiu J, Swensen J, Reddy S. High microsatellite instability (MSI-H) colorectal carcinoma: a brief review of predictive biomarkers in 
the era of personalized medicine. Fam Cancer. 2016 Jul;15(3):405-12. doi: 10.1007/s10689-016-9884-6.

Golob A, Gadžo D, Stibilj V, Djikić M, Gavrić T, Kreft I, Germ M. Sulphur interferes with selenium accumulation in Tartary buckwheat plants. Plant Physiol Biochem. 2016 Nov;108:32-36. doi: 10.1016/j. plaphy.2016.07.001. Epub 2016 Jul 4.

Hodžić A, Alić A, Prašović S, Otranto D, Baneth G, Duscher GG. Hepatozoon silvestris sp. nov.: morphological and molecular characterization of a new species of Hepatozoon (Adeleorina: Hepatozoidae) from the European wild cat (Felis silvestris silvestris). Parasitology. 2017 Apr;144(5):650-661. doi: 10.1017/ S0031182016002316. Epub 2016 Dec 12.

Hudić I, Szekeres-Bartho J, Stray-Pedersen B, Fatušić Z, Polgar B, Ećim-Zlojutro V. Lower Urinary and Serum Progesterone-Induced Blocking Factor in Women with Preterm Birth. J Reprod Immunol. 2016 Sep;117:66-9. doi: 10.1016/j.jri.2016.07.003. Epub 2016 Jul 25.

Ibrahimagić OĆ, Smajlović D, Dostović Z, Iljazović A, Kojić B, Zonić L. Neurobrucellosis and cerebral venous sinus thrombosis: a case report. Acta Clin Belg. 2016 Nov 2:1-3. [Epub ahead of print]

Ibrahimagić OĆ, Smajlović D, Dostović Z, Zvorničanin J, Pašić Z, Salihović Hajdarević D. Vogt-Koyanagi-Harada syndrome and hyperhomocysteinemia: Is there a hidden link? Med Hypotheses. 2017 Feb;99:47-48. doi: 10.1016/j. mehy.2016.12.009. Epub 2016 Dec 26.

Ivankovic S, Stojkovic R, Maksimovic M, Galic B, Milos M. Impact of calcium ion on cytotoxic effect of the boroxine derivative, K2[B3O3F4OH]. J Enzyme Inhib Med Chem. 2016;31(sup3):70-74. Epub 2016 Jul 7.

Jeon I, Zeljkovic S, Kondo K, Yoshizawa M, Matsuo Y. Interface Engineering of Metal Oxides using Ammonium Anthracene in Inverted Organic Solar Cells. ACS Appl Mater Interfaces. 2016 Nov 9;8(44):29866-29871. Epub 2016 Oct 27.

Jevremovic V. PS01.27: A Comparison Between Clinically Apparent Gastrointestinal Metastasis of a Primary Lung Carcinoma and the Incidence in Post-Mortem Studies: Topic: Medical Oncology. J Thorac Oncol. 2016 Nov;11(11S):S285. doi: 10.1016/j. jtho.2016.09.062. Epub 2016 Oct 28.

Jokić S, Rajić M, Bilić B, Molnar M. Supercritical Extraction of Scopoletin from Helichrysum italicum
(Roth) G. Don Flowers. Phytochem Anal. 2016 Sep;27(5):290-5. doi: 10.1002/pca.2630. Epub 2016 Jul 20.

Joneja U, Vranic S, Swensen J, Feldman R, Chen W, Kimbrough J, Xiao N, Reddy S, Palazzo J1, Gatalica Z. Comprehensive profiling of metaplastic breast carcinomas reveals frequent overexpression of programmed death-ligand 1. J Clin Pathol. 2017 Mar;70(3):255-259. doi: 10.1136/ jclinpath-2016-203874. Epub 2016 Aug 16.

Jovanovic N, Peek-Asa C, Swanton A, Young T, Alajbegovic-Halimic J, Cavaljuga S, Nisic F. Prevalence and risk factors associated with workrelated eye injuries in Bosnia and Herzegovina. Int J Occup Environ Health. 2016 Oct;22(4):325-332. Epub 2016 Nov 4.

Jovanović D, Šraml M, Matović B, Mićić S. An examination of the construct and predictive validity of the self-reported speeding behavior model. Accid Anal Prev. 2017 Feb;99(Pt A):66-76. doi: 10.1016/j. aap.2016.11.015. Epub 2016 Nov 22.

Jurišić S, Kozomara D, Jurić H, Verzak Ž, Jurišić G. The influence of different types of brackets and efficacy of two chlorhexidine mouthwashes on oral hygiene and the incidence of white spot lesions in adolescents during the orthodontic therapy. Psychiatr Danub. 2016 Dec;28(Suppl-2):247-252.

Karabuva S, Brizić I, Latinović Z, Leonardi A, Križaj I, Lukšić B. Cardiotoxic effects of the Vipera ammodytes ammodytes venom fractions in the isolated perfused rat heart. Toxicon. 2016 Oct;121:98104. doi: 10.1016/j.toxicon.2016.09.001. Epub 2016 Sep 11.

Karan Križanac D, Krasić Arapović A, Skočibušić S, Pintarić I, Trgo G, Tomić S.CD44 Immunoexpression is Unfavorable Predictor in Ovarian Serous Cancer. Appl Immunohistochem Mol Morphol. 2016 Aug 3. [Epub ahead of print]

Kocic M, Djuricic SM, Djordjevic M, Savic D, Kecman B, Sarajlija A. Appendiceal involvement in a patient with Gaucher disease. Blood Cells Mol Dis. 2016 Sep 30. pii: S1079-9796(16)30198-X. doi: 10.1016/j.bcmd.2016.09.002. [Epub ahead of print]

Kolar E, Pavletič MS, Smrdu M, Atiković A. Athletes' perception of the causes of injury in gymnastics. J Sports Med Phys Fitness. 2017 May;57(5):703-710. doi: 10.23736/S0022-4707.16.06228-9. Epub 2016 Mar 31. 
Koopman FA, Chavan SS, Miljko S, Grazio S, Sokolovic S, Schuurman PR, Mehta AD, Levine YA, Faltys M, Zitnik R, Tracey KJ, Tak PP. Vagus nerve stimulation inhibits cytokine production and attenuates disease severity in rheumatoid arthritis. Proc Natl Acad Sci U S A. 2016 Jul 19;113(29):82849. doi: 10.1073/pnas.1605635113. Epub 2016 Jul 5.

Kotseva K, De Bacquer D, De Backer G, Rydén L, Jennings C, Gyberg V, Abreu A, Aguiar C, Conde AC, Davletov K, Dilic M, Dolzhenko M, Gaita D, Georgiev B, Gotcheva N, Lalic N, Laucevicius A, Lovic D, Mancas S, Miličić D, Oganov R, Pajak A, Pogosova N, Reiner Ž, Vulic D, Wood D, On Behalf Of The Euroaspire Investigators. Lifestyle and risk factor management in people at high risk of cardiovascular disease. A report from the European Society of Cardiology European Action on Secondary and Primary Prevention by Intervention to Reduce Events (EUROASPIRE) IV cross-sectional survey in 14 European regions. Eur J Prev Cardiol. 2016 Dec;23(18):2007-2018. doi: 10.1177/2047487316667784. Epub 2016 Sep 27.

Kozomara D, Šutalo N, Galić G, Jurišić S, Trninć Z. Giant Unilateral Breast Juvenile Fibroadenoma - the Aesthetic outcome of Surgical Treatment Should Be Considered. Psychiatr Danub. 2016 Dec;28(Suppl-2):256-259.

Krleža-Jerić K, Gabelica M, Banzi R, Martinić MK, Pulido B, Mahmić-Kaknjo M, Reveiz L, Šimić J, Utrobičić A, Hrgović I. IMPACT Observatory: tracking the evolution of clinical trial data sharing and research integrity. Biochem Med (Zagreb). 2016 Oct 15;26(3):308-307.

Kurtcehajic A, Vele E, Hujdurovic A. Portal vein aneurysm and portal biliopathy. J Hepatobiliary Pancreat Sci. 2016 Oct;23(10):658. doi: 10.1002/ jhbp.383. Epub 2016 Aug 25.

Kurtcehajic A, Zerem E, Boričić I, Hujdurovic A, Zerem O. Multicentric Reticulohistiocytosis: A Unique Case of Chronic Colitis. Am J Gastroenterol. 2016 Sep;111(9):1227. doi: 10.1038/ajg.2016.150.

Kurtovic-Kozaric A, Mehinovic L, Malesevic R, Mesanovic S, Jaros T, Stomornjak-Vukadin M, Mackic-Djurovic M, Ibrulj S, Kurtovic-Basic I, Kozaric M. Ten-year trends in prevalence of Down syndrome in a developing country: impact of the maternal age and prenatal screening. Eur J Obstet Gynecol Reprod Biol. 2016 Nov;206:79-83. doi: 10.1016/j.ejogrb.2016.08.038. Epub 2016 Sep 12.

Lecic J, Cakic S, Janjic Pavlovic O, Cicmil A, Vukotic O, Petrovic V, Cicmil S. Different methods for subgingival application of chlorhexidine in the treatment of patients with chronic periodontitis. Acta Odontol Scand. 2016 Aug;74(6):502-7. doi: 10.1080/00016357.2016.1206964. Epub 2016 Jul 13.

Mahmic-Kaknjo M, Marusic A. Decentralized Decision Making On Drug Reimbursement In A Middle-Income Country: Case Of Bosnia And Herzegovina. Clin Ther. 2016 Oct 6;38(10S):e17. doi: 10.1016/j.clinthera.2016.07.126.

Mahmic-Kaknjo M, Marusic A. Use of Health Technology Assessment Agencies' Reports As Evidence For Making Decisions On Drug Reimbursement In Decentralized Decision-Making Environment. Clin Ther. 2016 Oct 6;38(10S):e30. doi: 10.1016/j.clinthera.2016.07.161.

Maksimović Z, De la Fe C, Amores J, Gómez-Martín Á, Rifatbegović M. Comparison of phenotypic and genotypic profiles among caprine and ovine Mycoplasma ovipneumoniae strains. Vet Rec. 2017 Feb 18;180(7):180. doi: 10.1136/vr.103699. Epub 2016 Nov 28.

Malic Z, Topic A, Francuski D, Stankovic M, Nagorni-Obradovic L, Markovic B, Radojkovic D. Oxidative Stress and Genetic Variants of Xenobiotic-Metabolising Enzymes Associated with COPD Development and Severity in Serbian Adults. COPD. 2017 Feb;14(1):95-104. doi: 10.1080/15412555.2016.1199667. Epub 2016 Jul 15.

Markotic F, Cerni Obrdalj E, Zalihic A, Pehar R, Hadziosmanovic Z, Pivic G, Durasovic S, Grgic V, Banozic A, Sapunar D, Puljak L. Adherence To Pharmacological Treatment Of Chronic NonMalignant Pain In Individuals Aged 65 and Older. Clin Ther. 2016 Oct 6;38(10S):e11. doi: 10.1016/j. clinthera.2016.07.111.

Martinovic V, Vukusic Pusic T, Restovic I, Bocina I, Filipovic N, Saraga-Babic M, Vukojevic K. Expression of Epithelial and Mesenchymal Differentiation Markers in the Early Human Gonadal Development. Anat Rec (Hoboken). 2016 Dec 16. doi: 10.1002/ ar.23531. [Epub ahead of print]

Mehić B, Duranović Rayan L, Bilalović N, Dohranović Tafro D, Pilav I. Lung adenocarcinoma mimicking pulmonary fibrosis-a case report. BMC Cancer. 2016 Sep 13;16(1):729. doi: 10.1186/s12885016-2763-6.

Memisevic H, Mujkanovic E, Ibralic-Biscevic I. Facial Emotion Recognition in Adolescents With Disabilities: The Effects of Type of Disability and 
Gender. Percept Mot Skills. 2016 Aug;123(1):127-37. doi: 10.1177/0031512516660781. Epub 2016 Jul 20.

Merhemic Z, Stosic-Opincal T, Thurnher MM. Neuroimaging of Spinal Tumors. Magn Reson Imaging Clin N Am. 2016 Aug;24(3):563-79. doi: 10.1016/j.mric.2016.04.007.

Mihailović DT, Drešković N, Arsenić I, Ćirić V, Djurdjević V, Mimić G, Pap I, Balaž I. Impact of climate change on soil thermal and moisture regimes in Serbia: An analysis with data from regional climate simulations under SRES-A1B. Sci Total Environ. 2016 Nov 15;571:398-409. doi: 10.1016/j. scitotenv.2016.06.142. Epub 2016 Jul 27.

Naganuma T, Mitomo S, Yabushita H, Kawamoto H, Nakao T, Lazarevic A, Nakamura S. Kissing balloon inflation in the aortic valve and left main stem: A novel coronary protection technique. Int J Cardiol. 2016 Nov 15;223:571-573. doi: 10.1016/j. ijcard.2016.08.187. Epub 2016 Aug 9.

Ostojic M, Soljic V, Vukojevic K, Dapic T. Immunohistochemical characterization of early and advanced knee osteoarthritis by NF- $\kappa \mathrm{B}$ and iNOS expression. J Orthop Res. 2016 Dec 13. doi: 10.1002/ jor.23504. [Epub ahead of print]

Pagani L, Lawson DJ, Jagoda E, Mörseburg A, Eriksson A, Mitt M, Clemente F, Hudjashov G, DeGiorgio M, Saag L, Wall JD, Cardona A, Mägi R, Sayres MA, Kaewert S, Inchley C, Scheib CL, Järve M, Karmin M, Jacobs GS, Antao T, Iliescu FM, Kushniarevich A, Ayub Q, Tyler-Smith C, Xue Y, Yunusbayev B, Tambets K, Mallick CB, Saag L, Pocheshkhova E, Andriadze G, Muller C, Westaway MC, Lambert DM, Zoraqi G, Turdikulova S, Dalimova D, Sabitov Z, Sultana GN, Lachance J, Tishkoff S, Momynaliev K, Isakova J, Damba LD, Gubina M, Nymadawa P, Evseeva I, Atramentova L, Utevska O, Ricaut FX, Brucato N, Sudoyo H, Letellier T, Cox MP, Barashkov NA, Škaro V, Mulahasanovic L, Primorac D, Sahakyan H, Mormina M, Eichstaedt CA, Lichman DV, Abdullah S, Chaubey G, Wee JT, Mihailov E, Karunas A, Litvinov S, Khusainova R, Ekomasova N, Akhmetova V, Khidiyatova I, Marjanović D, Yepiskoposyan L, Behar DM, Balanovska E, Metspalu A, Derenko M, Malyarchuk B, Voevoda M, Fedorova SA, Osipova LP, Lahr MM, Gerbault P, Leavesley M, Migliano AB, Petraglia M, Balanovsky O, Khusnutdinova EK, Metspalu E, Thomas MG, Manica A, Nielsen R, Villems R, Willerslev E, Kivisild T, Metspalu M. Genomic analyses inform on migration events during the peopling of Eurasia. Nature. 2016 Oct 13;538(7624):238-242. doi: 10.1038/nature19792. Epub 2016 Sep 21.
Pavlović M, Babić D, Rastović P, Babić R, Vasilj M. Metabolic Syndrome, Total and Differential White Blood Cell Counts in Patients with Schizophrenia. Psychiatr Danub. 2016 Dec;28(Suppl-2):216-222.

Peckova K, Martinek P, Pivovarcikova K, Vanecek T, Alaghehbandan R, Prochazkova K, Montiel DP, Hora M, Skenderi F, Ulamec M, Rotterova P, Daum O, Ferda J, Davidson W, Ondic O, Dubova M, Michal M, Hes O. Cystic and necrotic papillary renal cell carcinoma: prognosis, morphology, immunohistochemical, and molecular-genetic profile of 10 cases. Ann Diagn Pathol. 2017 Feb;26:23-30. doi: 10.1016/j.anndiagpath.2016.10.007. Epub 2016 Oct 20 .

Pleadin J, Frece J, Kudumija N, Petrović D, Vasilj V, Zadravec M, Škrivanko M, Perković I, Markov $\mathrm{K}$. Citrinin in cereals and feedstuffs coming from Croatia and Bosnia \& Herzegovina. Food Addit Contam Part B Surveill. 2016 Dec;9(4):268-274. Epub 2016 Jul 26.

Pleadin J, Vasilj V, Kudumija N, Petrović D, Vilušić M, Škrivanko M. Survey of T-2/HT-2 toxins in unprocessed cereals, food and feed coming from Croatia and Bosnia \& Herzegovina. Food Chem. 2017 Jun 1;224:153-159. doi: 10.1016/j. foodchem.2016.12.063. Epub 2016 Dec 22.

Rades D, Strojan P, Seidl D, Janssen S, Bajrovic A, Kazic N, Hakim SG, Wollenberg B, Schild SE. Radiochemotherapy for locally advanced squamous cell carcinoma of the head and neck: Higher-dose cisplatin every 3 weeks versus cisplatin/5-fluorouracil every 4 weeks. J Craniomaxillofac Surg. 2016 Sep;44(9):1436-40. doi: 10.1016/j.jcms.2016.06.033. Epub 2016 Jul 7.

Raguz MJ, Glamuzina DS, Tomic V, Mikulic I. Does the BMI of Expectant Mothers Influence the Concentration of C-Reactive Protein in Newborns in the Early Neonatal Period? Z Geburtshilfe Neonatol. 2016 Dec;220(6):251-256. doi: 10.1055/s-0042112463. Epub 2016 Oct 27.

Rucci A, Carletti C, Cravero W, Strbac B. Use of IAEA's phase-space files for virtual source model implementation: Extension to large fields. Phys Med. 2016 Aug;32(8):1030-3. doi: 10.1016/j. ejmp.2016.07.006. Epub 2016 Jul 14.

Sapcanin A, Cakal M, Imamovic B, Salihovic M, Pehlic E, Jacimovic Z, Jancan G.Herbicide and pesticide occurrence in the soils of children's playgrounds in Sarajevo, Bosnia and Herzegovina. Environ Monit Assess. 2016 Aug;188(8):450. doi: 10.1007/s10661-016-5463-4. Epub 2016 Jul 6. 
Sardanelli F, Aase HS, Álvarez M, Azavedo E, Baarslag HJ, Balleyguier C, Baltzer PA, Beslagic V, Bick U, Bogdanovic-Stojanovic D, Briediene R, Brkljacic B, Camps Herrero J, Colin C, Cornford E, Danes J, de Geer G, Esen G, Evans A, Fuchsjaeger MH, Gilbert FJ, Graf O, Hargaden G, Helbich TH, Heywang-Köbrunner SH, Ivanov V, Jónsson Á, Kuhl CK, Lisencu EC, Luczynska E, Mann RM, Marques JC, Martincich L, Mortier M, Müller-Schimpfle M, Ormandi K, Panizza P, Pediconi F, Pijnappel RM, Pinker K, Rissanen T, Rotaru N, Saguatti G, Sella T, Slobodníková J, Talk M, Taourel P, Trimboli RM, Vejborg I, Vourtsis A, Forrai G. Position paper on screening for breast cancer by the European Society of Breast Imaging (EUSOBI) and 30 national breast radiology bodies from Austria, Belgium, Bosnia and Herzegovina, Bulgaria, Croatia, Czech Republic, Denmark, Estonia, Finland, France, Germany, Greece, Hungary, Iceland, Ireland, Italy, Israel, Lithuania, Moldova, The Netherlands, Norway, Poland, Portugal, Romania, Serbia, Slovakia, Spain, Sweden, Switzerland and Turkey. Eur Radiol. 2016 Nov 2. [Epub ahead of print]

Sehic A, Tulek A, Khuu C, Nirvani M, Sand LP, Utheim TP. Regulatory roles of microRNAs in human dental tissues. Gene. 2017 Jan 5;596:9-18. doi: 10.1016/j.gene.2016.10.009. Epub 2016 Oct 8 .

Skenderi F, Chikha A, Ibisevic N, Tatarevic-Suko A, Kantardzic N, Vranic S.Skeletal Muscle Metastases from Squamous Cell Carcinoma of the Cervix: Report of Two Cases With Literature Review. Int J Gynecol Pathol. 2016 Jul 7. [Epub ahead of print]

Skocibusic S, Martinac M, Arapovic J, Grgic S, Nikolic J, Hasanagic D, Bevanda M, Ravlija J. HBV and HCV serological monitoring among injection drugs users in opiate substitution treatment in Bosnia and Herzegovina. J Infect Dev Ctries. 2016 Sep 30;10(9):968-972. doi: 10.3855/jidc.7445.

Smits A, Kulo A, van den Anker J, Allegaert K. The amikacin research program: a stepwise approach to validate dosing regimens in neonates. Expert Opin Drug Metab Toxicol. 2017 Feb;13(2):157-166. doi: 10.1080/17425255.2017.1234606. Epub 2016 Sep 21.

Sokolovic S, Alimanovic-Alagic R, Dzananovic L, Cavaljuga S, Beslic N, Ferhatbegovic-Opankovic E. Vitamin D status in Bosnia and Herzegovina: the cross-sectional epidemiological analysis. Osteoporos Int. 2017 Mar;28(3):1021-1025. doi: 10.1007/s00198016-3831-0. Epub 2016 Nov 26.

Sokolovic S, Mehmedagic S. OS 11-06 The effect of vagus nerve stimulation on arterial hypertension using active implantable device. J Hypertens. 2016 Sep;34 Suppl 1 - ISH 2016 Abstract Book:e75.

Stanetic BM, Ostojic M, Campos CM, Marinkovic J, Farooq V, Kovacevic-Preradovic T, Huber K, Serruys PW. ApPropriateness of myocaRdial RevascularizatiOn assessed by the SYNTAX score II in a coUntry without cardiac Surgery faciliTies; PROUST study. Int J Cardiol. 2017 Jan 15;227:478484. doi: 10.1016/j.ijcard.2016.11.001. Epub 2016 Nov 3.

Stevanović NR, Perušković DS, Gašić UM, Antunović VR, Lolić AĐ, Baošić RM. Effect of substituents on prediction of TLC retention of tetra-dentate Schiff bases and their Copper(II) and Nickel(II) complexes. Biomed Chromatogr. 2017 Mar;31(3). doi: 10.1002/ bmc.3810. Epub 2016 Sep 7.

Talić G, Ostojić L, Bursać SN, Nožica-Radulović T, Stevanović-Papić Đ. Idiopathic Scoliosis from Psychopathological and Mind-Body Medicine Perspectives. Psychiatr Danub. 2016 Dec;28(4):357362.

Thielmann A, Gerasimovska-Kitanovska B, Buczkowski K, Koskela TH, Mevsim V, Czachowski S, Petrazzuoli F, Petek-Šter M, Lingner H, Hoffman RD, Tekiner S, Chambe J, Edirne T, Hoffmann K, Pirrotta E, Uludağ A, Yikilkan H, Kreitmayer Pestic S, Zielinski A, Guede Fernández C, Weltermann B. SelfCare for Common Colds by Primary Care Patients: A European Multicenter Survey on the Prevalence and Patterns of Practices-The COCO Study. Evid Based Complement Alternat Med. 2016;2016:6949202. Epub 2016 Sep 21.

Tica Sedlar I, Petricevic J, Saraga-Babic M, Pintaric I, Vukojevic K. Apoptotic pathways and stemness in the colorectal epithelium and lamina propria mucosae during the human embryogenesis and carcinogenesis. Acta Histochem. 2016 Sep;118(7):693-703. doi: 10.1016/j.acthis.2016.08.004. Epub 2016 Sep 6.

Tubić B, Uzunović A, Pilipović S, Gagić

Ž. Dissolution Profile of Nimesulide from Pharmaceutical Preparations for Oral Use. Acta Chim Slov. 2016;63(1):193-9.

van Bokhorst LG, Knapová L, Majoranc K, Szebeni ZK, Táborský A, Tomić D, Cañadas E. “It's Always the Judge's Fault": Attention, Emotion Recognition, and Expertise in Rhythmic Gymnastics Assessment. Front Psychol. 2016 Jul 5;7:1008. doi: 10.3389/ fpsyg.2016.01008. eCollection 2016.

Vele E, Kurtcehajic A, Zerem E, Maskovic J, Alibegovic E, Hujdurovic A. Plasma D-dimer 
as a predictor of the progression of abdominal aortic aneurysm. J Thromb Haemost. 2016 Nov;14(11):2298-2303. doi: 10.1111/jth.13487. Epub 2016 Oct 19.

Vrdoljak E, Bodoky G, Jassem J, Popescu RA, Mardiak J, Pirker R, Čufer T, Bešlija S, Eniu A, Todorović V, Kubáčková K, Kurteva G, Tomašević Z, Sallaku A, Smichkoska S, Bajić Ž, Šikić BI. Cancer Control in Central and Eastern Europe: Current Situation and Recommendations for Improvement. Oncologist. 2016 Oct;21(10):1183-1190. Epub 2016 Jul 8.

Yusuf E, Ong DS, Martin-Quiros A, Skevaki C, Cortez J, Dedić K, Maraolo AE, Dušek D, Maver PJ, Sanguinetti M, Tacconelli E; Trainee Association of the European Society of Clinical Microbiology and Infectious Diseases (ESCMID). A large survey among European trainees in clinical microbiology and infectious disease on training systems and training adequacy: identifying the gaps and suggesting improvements. Eur J Clin Microbiol Infect Dis. 2017 Feb;36(2):233-242. doi: 10.1007/s10096-016-2791-9. Epub 2016 Oct 4.

Zhou K, Yee SW, Seiser EL, van Leeuwen N, Tavendale R, Bennett AJ, Groves CJ, Coleman RL, van der Heijden AA, Beulens JW, de Keyser CE, Zaharenko L, Rotroff DM, Out M, Jablonski KA, Chen L, Javorský M, Židzik J, Levin AM, Williams LK, Dujic T, Semiz S, Kubo M, Chien HC, Maeda S, Witte JS, Wu L, Tkáč I, Kooy A, van Schaik RH,
Stehouwer CD, Logie L; MetGen Investigators; DPP Investigators; ACCORD Investigators, Sutherland C, Klovins J, Pirags V, Hofman A, Stricker BH, Motsinger-Reif AA, Wagner MJ, Innocenti F, Hart LM, Holman RR, McCarthy MI, Hedderson MM, Palmer CN, Florez JC, Giacomini KM, Pearson ER. Variation in the glucose transporter gene SLC2A2 is associated with glycemic response to metformin. Nat Genet. 2016 Sep;48(9):1055-9. doi: 10.1038/ng.3632. Epub 2016 Aug 8.

Zielinski C, Láng I, Inbar M, Kahán Z, Greil R, Beslija S, Stemmer SM, Zvirbule Z, Steger GG, Melichar B, Pienkowski T, Sirbu D, Petruzelka L, Eniu A, Nisenbaum B, Dank M, Anghel R, Messinger D, Brodowicz T; TURANDOT investigators.

Bevacizumab plus paclitaxel versus bevacizumab plus capecitabine as first-line treatment for HER2negative metastatic breast cancer (TURANDOT): primary endpoint results of a randomised, openlabel, non-inferiority, phase 3 trial. Lancet Oncol. 2016 Sep;17(9):1230-9. doi: 10.1016/S14702045(16)30154-1. Epub 2016 Aug 5.

Žunić ZS, Bossew P, Bochicchio F, Veselinovic N, Carpentieri C, Venoso G, Antignani S, Simovic R, Ćurguz Z, Udovicic V, Stojanovska Z, Tollefsen T. The relation between radon in schools and in dwellings: A case study in a rural region of Southern Serbia. J Environ Radioact. 2017 Feb;167:188-200. doi: 10.1016/j.jenvrad.2016.11.024. Epub 2016 Dec 3.

by Nerma Tanović 